
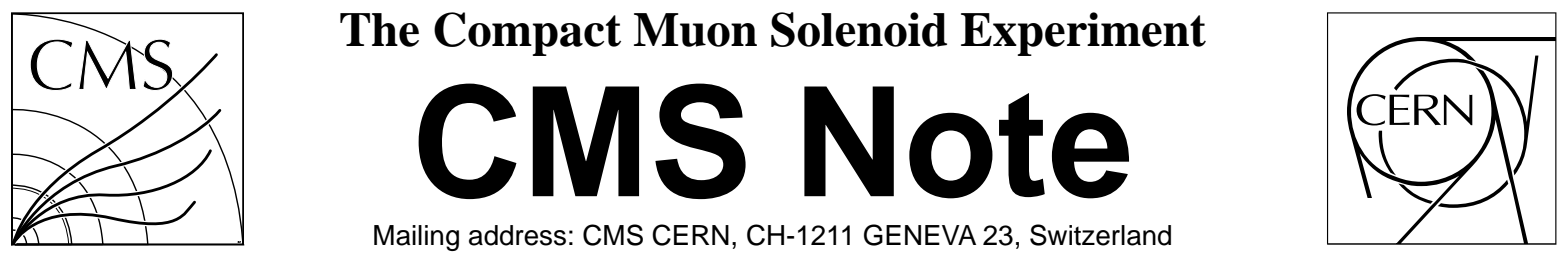

22 May 2006

\title{
Study of Pair-Produced Doubly Charged Higgs Bosons with a Four Muon Final State at the CMS Detector
}

\author{
T. Rommerskirchen and T. Hebbeker
}

RWTH Aachen, III. Phys.Inst. A, Aachen, Germany

\begin{abstract}
An analysis of the discovery potential of doubly charged Higgs bosons pair-produced in Drell-Yan events at the CMS detector is presented in this note. The decay branching ratio into muon pairs is assumed to be $100 \%$. The pure muonic decay channel yields a clear signal which is almost background free. Doubly charged Higgs bosons with masses in the range 100 to $800 \mathrm{GeV}$ are studied, for a low luminosity scenario of $\mathcal{L}=2 \times 10^{33} \mathrm{~cm}^{-2} \mathrm{~s}^{-1}$. The full detector simulation is used. Doubly charged Higgs bosons in this production and decay channel with masses $m_{H^{ \pm \pm}} \leq 650 \mathrm{GeV}$ are expected to be observable at CMS with a significance exceeding $5 \sigma$ at $10 \mathrm{fb}^{-1}$ of integrated luminosity. If no signal will be detected for this integrated luminosity, the existence of a doubly charged Higgs boson with $m_{H^{ \pm \pm}} \leq 760 \mathrm{GeV}$ can be excluded at $95 \%$ Confidence level. This exceeds the current exclusion limit $m_{H^{ \pm \pm}} \leq 136 \mathrm{GeV}$, set by CDF at Tevatron Run II, by $624 \mathrm{GeV}$.
\end{abstract}




\section{Introduction}

One of the main goals of the future CMS detector is to find or exclude the Standard Model Higgs boson. The Higgs mechanism gives mass to $\mathrm{W}$ and $\mathrm{Z}$ bosons as well as to quarks and leptons by introducing two new fields in a complex scalar isopin doublet $(\mathrm{I}=1 / 2, \mathrm{Y}=1)$, which has a non-zero vacuum expectation value. This non-zero vacuum expectation value breaks the electroweak symmetry. Particles gain their mass by coupling to the Higgs field. The coupling connects left handed and right handed fermion fields. As there are no right handed neutrinos in the Standard Model, neutrinos do not couple to the Higgs field and therefore gain no mass.

Recent neutrino oscillation experiments however show that neutrinos have a tiny but nonzero mass [1] [3]. The Standard Model needs to be extended to explain neutrino masses.

Possible extensions are Higgs triplet models $(\mathrm{I}=1, \mathrm{Y}=2)$. Higgs triplet models could explain the non-zero neutrino mass, either by introducing the "see-saw" mechanism as in left-right symmetric models [5], or by direct Yukawa coupling of the Higgs bosons to neutrinos [9]. Higgs boson triplets couple to leptons according to[5]:

$$
\mathcal{L}_{Y}=h_{i j} \Psi_{i L}^{T} C \sigma_{2} H \Psi_{j L}+h . c .,
$$

where $\mathrm{H}$ is the Higgs triplet $\left(H^{0}, H^{+}, H^{++}\right), \Psi_{L}$ is a left handed lepton doublet $\Psi_{i L}=\left(\nu_{i L}, l_{i L}\right), i, j$ are flavour indices, $\mathrm{C}$ is the Dirac charge conjugation operator and $h_{i j}$ is the Yukawa coupling to leptons.

The Yukawa Lagrangian (1) leads to:

$$
m_{i j} \sim h_{i j}\left\langle H^{0}\right\rangle
$$

Where $\left\langle H^{0}\right\rangle$ is the vacuum-expectation value of the neutral Higgs triplet member. So the coupling of $H^{0}$ to neutrinos would give non-vanishing neutrino masses.

The detection of doubly charged Higgs bosons would be a clear signature of Higgs triplet models.

Doubly charged Higgs bosons couple directly to leptons, photons, W and Z bosons and charged Higgs bosons, but not to quarks. Possible lowest order production processes at Hadron Colliders are shown in Figure 1 and Figure 2.

The Standard Model Higgs mechanism predicts the $\mathrm{W}$ and $\mathrm{Z}$ boson masses to be at lowest order: $m_{W}^{2}=$

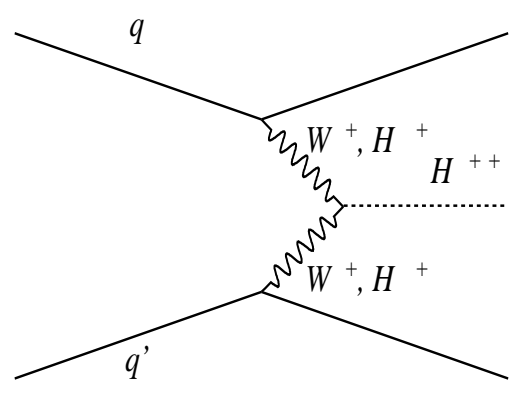

Figure 1: Single production of doubly charged Higgs bosons

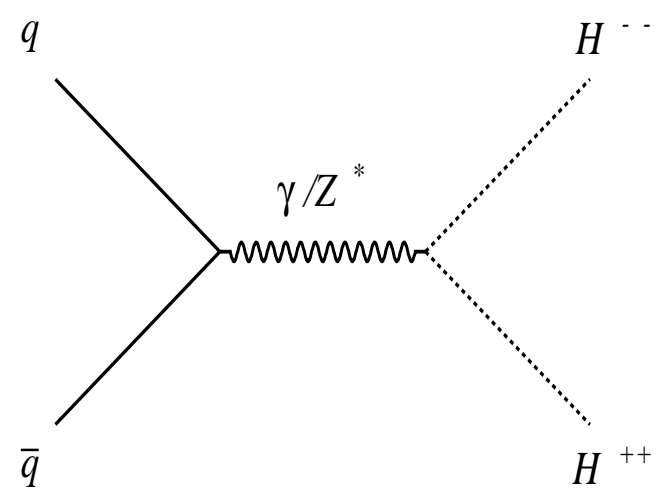

Figure 2: Drell-Yan pair production of doubly charged Higss Bosons

$m_{Z}^{2} \cos ^{2} \theta_{W}$, where $\theta_{W}$ is the electroweak mixing angle. This relation has been confirmed with great precision. This prediction is preserved if the vacuum expectation value of the neutral member of the Higgs triplet is very small. This constrains the cross section for the generation of a doubly charged Higgs by $W W H^{ \pm \pm}$vertex.

The production cross section by a $H^{\mp} H^{\mp} H^{ \pm \pm}$vertex or by a $W H^{\mp} H^{ \pm \pm}$vertex depends on the mass of the singly charged Higgs boson.

In this analysis pair-produced doubly charged Higgs bosons (Fig.2) are studied. The pair-production cross section depends only on the mass of the doubly charged Higgs boson.

Figure 3 shows the comparison between the cross section for pairproduction of doubly charged Higg Bosons and the cross section for the Standard Model process $H \rightarrow Z \bar{Z} \rightarrow 4 \mu$ [6]. Both cross sections are the same order of magnitude. Doubly charged Higgs bosons decay into equally charged particles, therefore the signal is clearly distinguishable from many Standard Model background processes. 


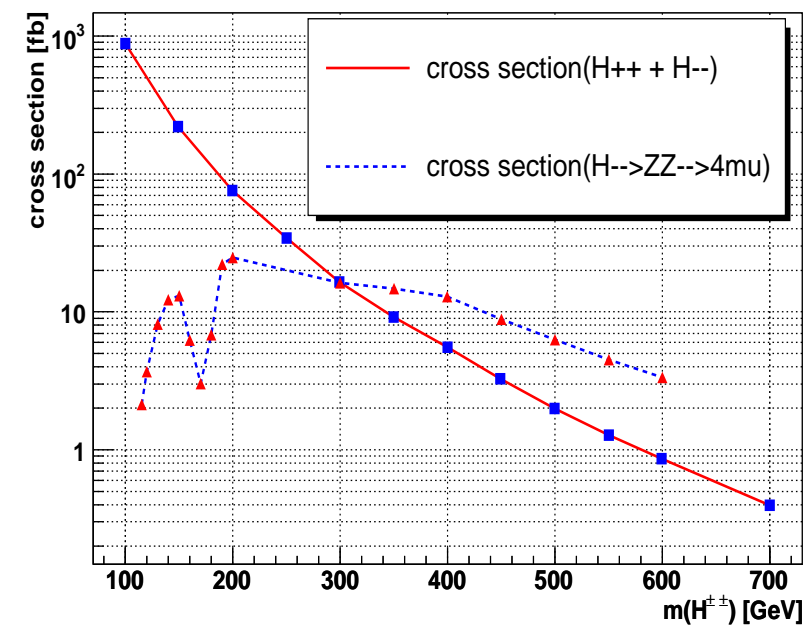

Figure 3: Cross section for pairproduction of $H^{ \pm \pm}$compared to the cross section for $H \rightarrow Z \bar{Z} \rightarrow 4 \mu$ (cross section times branching ratios)

In this note the $\mathrm{H}^{++} \mathrm{H}^{--} \rightarrow 4 \mu$ decay mode was investigated. Leptonic decay modes dominate in most Higgs triplet models and a pure muonic final state yields a signal which is almost background free.

The coupling of the triplet Higgs bosons to muons, $h_{\mu \mu}$, is constrained by the accurate measurement of $(g-2)_{\mu}$ and the condition of vacuum stability. The current limit obtained from the anomalous magnetic momentum is [5]:

$$
h_{\mu \mu}^{2} \leq 2.5 \cdot 10^{-5} \mathrm{GeV}^{-2} \cdot m_{H^{++}}^{2}
$$

The limit from the conditions of vacuum stability is independent of the Triplet Higgs mass [10] : $h_{\mu \mu} \leq 1.2$

For this study $h_{\mu \mu}=0.1$ was assumed. This coupling constant is large enough to force the generated doubly charged Higgs bosons to decay inside the CMS-Tracker. The $100 \%$ branching ratio into muons was chosen arbitrarily, and can easily be rescaled for other branching ratios in different Higgs triplet models.

Direct searches at the CDF experiment during the Tevatron Run II exclude the existence of doubly charged Higgs bosons in the $H^{++}+H^{--} \rightarrow 4 \mu$ channel for $m_{H^{ \pm \pm}} \leq 136 \mathrm{GeV}$ [4].

This note starts with an introduction to the muon detection in the CMS detector, which is crucial for this analysis with its four muon final state. In the next section the event generation and detector simulation will be described. The further sections deal with the several online and offline selection cuts and the statistical interpretation of the final results.

\section{Detection of Muons in CMS}

The accurate description of the CMS detector is given in [2]. Details are given here about the CMS muons detector, which is crucial for this study.

The CMS muon system [19] is interleaved with the iron return yoke needed for the $4 \mathrm{~T}$ coil and consists of three detection subsystems: drift-tube chambers (DT) in the barrel part $(|\eta|<1.2)$, cathode strip chambers (CSC) in the two end-caps $(1.2<|\eta|<2.4)$ and resistive plate chambers (RPC) in both barrel and end-caps. A muon originating from the nominal $p p$ interaction point crosses up to four muon stations.

Each drift tube chamber in the barrel contains $3 \times 4$ layers of staggered drift cells, where $2 \times 4$ layers measure the bending of the muon trajectory in the $(r, \phi)$-plane of CMS while one group of 4 layers determines the coordinate perpendicular to it $(\theta$ view). The only exception of this scheme are the outermost chambers consisting of only two superlayers in the $(r, \phi)$-plane. The basic detection element of a drift tube chamber in the barrel region is a $42 \times 13$ $\mathrm{mm}$ drift cell, flushed with a gas mixture of $85 \% \mathrm{Ar}$ and $15 \% \mathrm{CO}_{2}$.

The cathode strip chambers in both end-caps are multi-wire proportional chambers $\left(40 \% \mathrm{Ar}, 50 \% \mathrm{CO}_{2}, 10 \% \mathrm{CF}_{4}\right)$ with six individual layers of anode wires and segmented cathodes. The strips inside the trapezoidal chambers are oriented radially with the wires perpendicular to them. Strips measure the muon position in the azimuthal $\phi$ 
direction and wires provide, less precisely but with faster response, the radial position. The fast wire information is primarily used for the trigger but supports also the coordinate reconstruction although the high spatial precision is provided by the cathode information.

The resistive plate chamber system is complementary to the other muon detectors: with their reasonable spatial resolution and excellent time resolution of few nanoseconds, they are specifically designed for trigger purposes and add robustness and redundancy to the muon system.

The CMS trigger system has two levels, where the first level (L1) is implemented on custom-built hardware and the second level (HLT) is based on software. The first level global trigger has to decide every 25 ns whether to accept or reject the event based on coarse information from the calorimeter and the muon system (Global Muon Trigger).

The Global Muon Trigger may use information from all three complementary subsystems - the fast RPC timing information, the regional CSC and regional DT trigger - with the goal of reconstructing position and momentum of muons and assigning a bunch crossing with high efficiency.

The L1 muon trigger for DT and CSC works in several consecutive steps. First, the information of each chamber is processed independently by on-chamber local trigger electronics to reconstruct track segments at the chamber level. The individual segments are matched by higher level trigger electronics into a single muon candidate, assigning the track parameters $p_{T}, \eta, \phi$ and quality. The Global Muon Trigger matches the muon candidates from the DT and CSC Track Finder with the RPC information in order to choose the four best muon candidates with the highest transverse momentum $p_{T}$.

The CMS tracker provides up to 10 measurements of the track trajectory using silicon strip detectors. The muon momentum is measured with a high resolution through bending in a $4 \mathrm{~T}$ magnetic field. The full muon reconstruction is achieved by combining the silicon tracker information with the measurements in the muon system. The tracker dominates the momentum resolution for muon momenta up to $\sim 200 \mathrm{GeV}$, due to the fact that multiple scattering in the iron return yoke limits the stand-alone momentum resolution of the muon system.

\section{Event Generation and Detector Simulation}

Both signal and background event samples were generated at the Leading Order (LO) approximation; Next to Leading Order (NLO) production cross-sections, computed using different methods, are used for their normalization.

\subsection{Signal}

Signal event samples were generated using the event generator PYTHIA 6.227 [11] with CMKIN 4_3_1[13]. A collection of signal datasets were produced for $m_{H^{ \pm}}$between $100 \mathrm{GeV}$ and $800 \mathrm{GeV}$. Table 1 shows the number of events generated for the different signal data samples before pre-selection.

Table 1: Number of events generated with PYTHIA for different Signal Data samples, before any pre-selection.

\begin{tabular}{|c|cccccccc|}
\hline$m_{H^{ \pm \pm}}$in $\mathrm{GeV}$ & 200 & 300 & 400 & 600 & 650 & 700 & 750 & 800 \\
\hline Number of generated events & 8832 & 9000 & 1350 & 2500 & 5000 & 4840 & 4860 & 5000 \\
\hline
\end{tabular}

The generated events were processed through a full simulation of the CMS detector using OSCAR [15]. This was followed by digitization and superposition of simulated pileup events at low luminosity $\left(2 \times 10^{33} \mathrm{~cm}^{-2} \mathrm{~s}^{-1}\right)$. Finally reconstruction of physics objects was performed by using ORCA (Object-oriented Reconstruction for CMS Analysis) [14].

\subsection{Background}

Important backgrounds with a four muon final state are:

- $p p \rightarrow t \bar{t} \rightarrow W^{+} W^{-} b \bar{b} \rightarrow 2 \mu+2 \mu+X$ (generated with PYTHIA).

- $p p \rightarrow\left(Z^{(*)} / \gamma^{*}\right) \bar{b} b \rightarrow 2 \mu+2 \mu+X$ (generated with CompHEP [12]) 
- $\left.p p \rightarrow\left(Z^{(*)} / \gamma\right)\left(Z^{(*)}\right) / \gamma\right) \rightarrow 2 \mu+2 \mu$ (generated with CompHEP)

- $p p \rightarrow\left(Z^{(*)} / \gamma\right)\left(Z^{(*)} / \gamma\right) \rightarrow 2 \tau+2 \mu$ (generated with CompHEP)

The background contribution from $b \bar{b}$ production, which yields the highest probabilty to fake events with multiple muons was also investigated. It was found that the $b \bar{b}$ QCD background can be neglected after the online selection (Section 5) by requiring 4 well-reconstructed muons with pseudorapidity $|\eta|<2.1$ and transverse momentum $p_{T}>8 \mathrm{GeV}$.

The $t \bar{t}$ event sample was generated using PYTHIA with CMKIN 1_1_0 including LO processes $g g \rightarrow t \bar{t}$ and $q \bar{q} \rightarrow t \bar{t}$. The $\mathrm{W}$ bosons in the $t \bar{t}$ sample were forced to decay into electrons, muons and taus. The tau leptons were forced to decay into electrons and muons.

The $Z \bar{b} b$ and the $Z Z$ backgrounds originate either from gluons or quarks in the initial state. The $Z \bar{b} b$ sample was generated using CompHEP 4.2 and PYTHIA with CMKIN 3_1_0, including the matrix element generator for both initial states decaying into $\mu^{+} m u^{-} b \bar{b}$. The $\mathrm{Z}$ boson in the $Z \bar{b} b$ sample was generated with $m_{Z / \gamma *}>5 \mathrm{GeV}$ and is forced to decay into muons. The $\mathrm{b}$ and $\bar{b}$ quarks decay freely.

For the ZZ background sample only the $q \bar{q}$ distribution is generated, as neither PYTHIA nor compHEP include the process $g g \rightarrow\left(Z^{(*)} / \gamma^{*}\right)\left(Z^{(*)} / \gamma^{*}\right)$. The LO generation was done using CompHEP as it includes both, $\mathrm{t}$ - and schannel diagrams. In the $Z Z \rightarrow 4 \mu$ sample both $Z$ bosons were forced to decay into muons. In the $Z Z \rightarrow 2 \tau+2 \mu$ sample one $\mathrm{Z}$ boson was forced to decay muonically one was forced to decay into taus. The muons generated in $\mathrm{Z}$ decays were forced to be generated with $p_{T}>3$ and $|\eta|<2.5$. The taus decay freely.

The detector simulation of the background samples is done using OSCAR and ORCA.

\subsection{Generator Level Pre-selection}

The following pre-selection requirements were made on all samples:

- Final state containing two positive and two negative muons;

- Transverse momentum $p_{T}(\mu)>3 \mathrm{GeV}$ and pseudorapidity $|\eta(\mu)|<2.4$ for all muons.

In the $Z \bar{b} b$ and the $Z Z$ sample it is also required that $5 \mathrm{GeV}<m\left(\mu^{+}+\mu^{-}\right)<150 \mathrm{GeV}$ for two of the possible combinations of muons. The effect of this cut however is negligible for the study described in this note. The forced tau decay in the $t \bar{t}$ sample leads to wrong pre-selection efficiencies, as PYTHIA uses wrong branching ratios for this forced decay mode. Each selected event has to be reweighted in order to get the correct pre-selection efficiency of each event.

The number of generated events in the background Monte Carlo data samples, after pre-selection, can be found in Table 2.

Table 2: Number of generated events in the background Monte Carlo data samples, after pre-selection

\begin{tabular}{|c|cccc|}
\hline Background & $Z Z \rightarrow 4 \mu$ & $Z Z \rightarrow 2 \mu+2 \tau$ & $Z b b \rightarrow 4 \mu$ & $t \bar{t} \rightarrow 4 \mu$ \\
\hline Number of selected events & 118000 & 10000 & 112500 & 95000 \\
\hline
\end{tabular}

\section{Production Cross Section and Pre-selection Efficiency}

\subsection{Signal}

The LO and NLO cross sections [6] for the doubly charged Higgs boson production in Drell-Yan events are shown in Figure 4. The generated signal samples are normalized to the value of the total cross section at NLO [6].

The NLO cross section corrections are standard QCD corrections, with virtual gluon exchange, gluon emission and quark emission. The NLO corrections increase the cross section by about $20-30 \%$ with a total theoretical uncertainty of $10-15 \%$. Table 3 shows the pre-selection efficiency (see Section 3.3) for several doubly charged Higgs boson masses. It can be seen that the pre-selection efficiency improves with increasing mass. This is a consequence of the $p_{T}$ cut in the pre-selection since larger doubly charged Higgs boson masses correspond to larger transverse momentum of the decay products. 


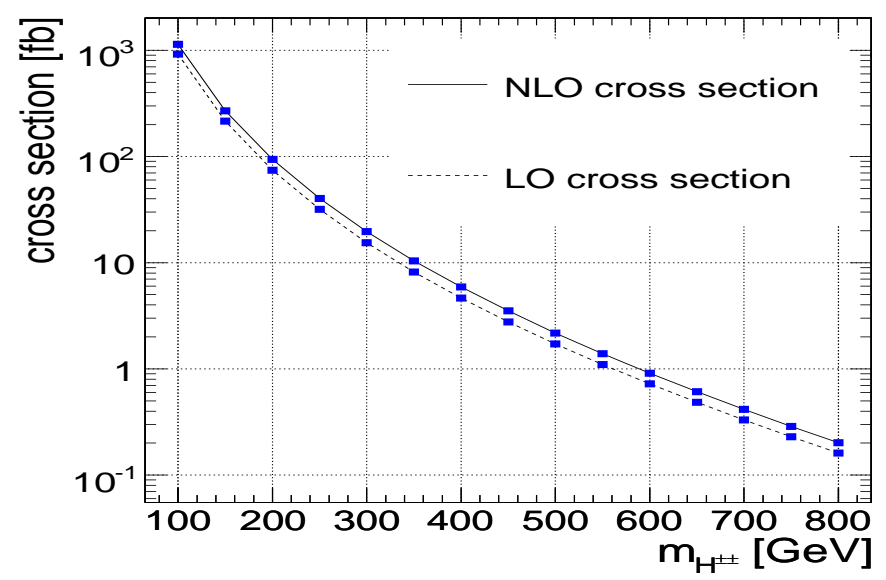

Figure 4: Cross section for pairproduction of $H^{ \pm \pm}$in LO and NLO

Table 3: Pre-selection efficiency, $\epsilon$, for signal datasamples with $\mathrm{m}\left(H^{ \pm \pm}\right)=200,300$ and $800 \mathrm{GeV}$, the errors are statistical only.

\begin{tabular}{|c|ccc|}
\hline$m_{H^{ \pm \pm}}$in $\mathrm{GeV}$ & 200 & 300 & 800 \\
\hline pre-selection efficiency in $\%$ & $85 \pm 1$ & $89 \pm 1$ & $94 \pm 2$ \\
\hline
\end{tabular}

\subsection{Background}

For the $t \bar{t}$ sample the NLO total cross section [7] is ( $840 \pm 5 \%$ scale $\pm 3 \%$ pdf)pb. For the $Z \bar{b} b$ sample the NLO order cross section, $278.4 \mathrm{pb}$, is obtained by scaling the LO value provided by CompHEP $116 \mathrm{pb}$ with a K-factor, $K_{N L O}=2.4 \pm 0.3$, calculated with MCFM [16]. The LO cross sections for $Z Z \rightarrow 4 \mu$ and $Z Z \rightarrow 2 \mu 2 \tau$ are respectively $113 \mathrm{fb}$ and $157 \mathrm{fb}$. The NLO cross section for the ZZ backgrounds is scaled by means of a K factor, $K_{N L O}=1.35$ [8]. It is evaluated for the t-channel contribution with MCFM and MadGraph [17].

The NLO cross section and the NLO cross section times branching ratio times pre-selection efficiency is shown in Table 4 for the different background data samples.

Table 4: NLO cross section and cross section times branching ratio times pre-selection efficiency for background datasamples, the errors are statistical only

\begin{tabular}{|c|cccc|}
\hline backgrounds & $Z Z \rightarrow 4 \mu$ & $Z Z \rightarrow 2 \mu+2 \tau$ & $Z b b \rightarrow 4 \mu$ & $t \bar{t} \rightarrow 4 \mu$ \\
\hline NLO cross-section $\sigma[\mathrm{fb}]$ & 153 & 212 & $278 \cdot 10^{3}$ & $840 \cdot 10^{3}$ \\
$\sigma \cdot B R \cdot \epsilon[\mathrm{fb}]$ & $87.4 \pm 0.3$ & $1.63 \pm 0.02$ & $290 \pm 0.9$ & $232 \pm 1$ \\
\hline
\end{tabular}

\section{Online Selection}

We request the events to satisfy both the Level 1 Di-Muon Trigger $(|\eta| \leq 2.1)$ and the High Level Di-Muon Trigger. Since four isolated muons are present in the final state, high triggering efficiencies are expected. Since the detector simulation uses the start-up detector geometry, the CSCs close to the beam pipe (ME1/1) have only a limited readout. This implies that the muon trigger is restricted to $|\eta|<2.1$.

The High-Level Di-Muon Trigger requires an event to contain at least two muons with $p_{T}>7 \mathrm{GeV}$, at least one has to be isolated in the Tracker. The $p_{T}$ sum of tracks contained in a cone around the muon direction is requested to be smaller than $0.97 \mathrm{GeV}$ ([14]). Further selection criterias for the High-Level-Muon Trigger are described in [18]. The L1+HLT efficiency for the signal samples after pre-selection is $>99 \%$ within uncertainties. The L1+HLT efficiency for the background is in average $70 \%$.

The lower trigger efficiency for the background is due to the isolation requirement which rejects some of the background containing non-isolated muons. The $p_{T}$ requirement also rejects some background, because most of the muons have low $p_{T}$. 


\section{Offline Reconstruction}

The offline reconstruction of muons is performed using the Global Muon Reconstructor. The Global Muon Reconstructor matches track segments reconstructed in the muon chambers with hits in the tracker system and performs a new fit.

Figure 5 shows the reconstruction efficiency for muons as a function of $\eta$ for the signal data sample $\left(m_{H^{ \pm \pm}}=\right.$ $300 \mathrm{GeV}$ ). The efficiency drops when one wheel ends and the next one begins. Nevertheless the overall scale is always $\geq 87 \%$. The global muon reconstructor reconstructs muons with $|\eta|<2.4$.

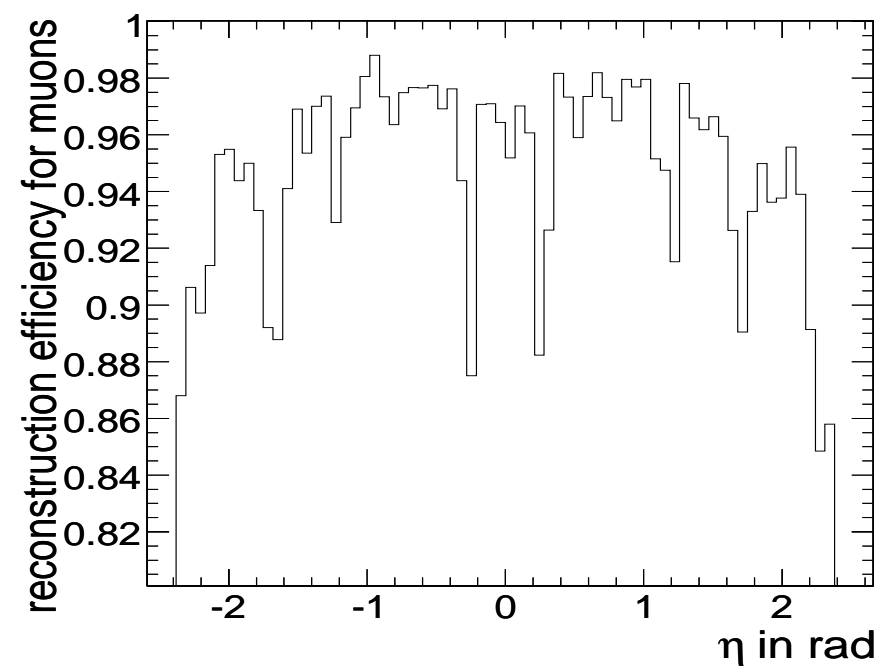

Figure 5: reconstruction efficiency for muons in dependency on $\eta$

\subsection{Impact Point}

The muon trajectory is extrapolated to the beamline. The point of closest approach to the origin of the detector coordinate system is defined as Impact Point [14].

\subsection{Reconstructed Doubly Charged Higgs Boson Mass}

The reconstructed muons are divided into positive and negative charged muons and sorted with increasing $p_{T}$. The invariant masses of the two highest $p_{T}$ muons with positive charge and of the two highest $p_{T}$ muons with negative charge are calculated.

Figure 6 shows the invariant mass for two signal samples and for the four background data samples. The invariant mass distributions of the backgound samples have been added up. All distributions are scaled to an integrated luminosity of $10 \mathrm{fb}^{-1}$.

The detector resolution, $\sigma_{d e t}$, for the reconstructed doubly charged Higgs boson mass has been estimated by using:

$$
\sigma_{r e c}^{2}=\sigma_{g e n}^{2}+\sigma_{d e t}^{2} \Rightarrow \sigma_{d e t}=\sqrt{\sigma_{r e c}^{2}-\sigma_{g e n}^{2}}
$$

where $\sigma_{r e c}$ is the width of the reconstructed mass distribution and $\sigma_{g e n}$ is the invariant mass of two highest $p_{T}$ generated muons with the same charge. By assuming this distribution to be gaussian the width $\sigma_{g e n}$ has been calculated. This width is determined by the width of the Breit-Wigner distribution of the doubly charged Higgs boson, radiative corrections and wrongly combined muons. It ranges from $\sim 4.5 \mathrm{GeV}$ at $m_{H^{ \pm \pm}}=200 \mathrm{GeV}$ to $\sim 5.5 \mathrm{GeV}$ at $m_{H^{ \pm \pm}}=800 \mathrm{GeV}$. Figure 7 shows the corresponding $\sigma_{\text {det }}$ as a function of the doubly charged Higgs boson mass.

The detector resolution worsens for increasing $m_{H^{ \pm} \pm}$. This can be explained by the worsening of the muon $p_{T}$ resolution with increasing $p_{T}: \frac{\Delta p_{T}}{p_{T}} \sim p_{T}$ [19]. Increasing doubly charged Higgs boson mass is correlated to increasing muon $p_{T}$. The detector resolution gives an estimate of the accuracy achievable on $m_{H^{ \pm \pm}}$. 

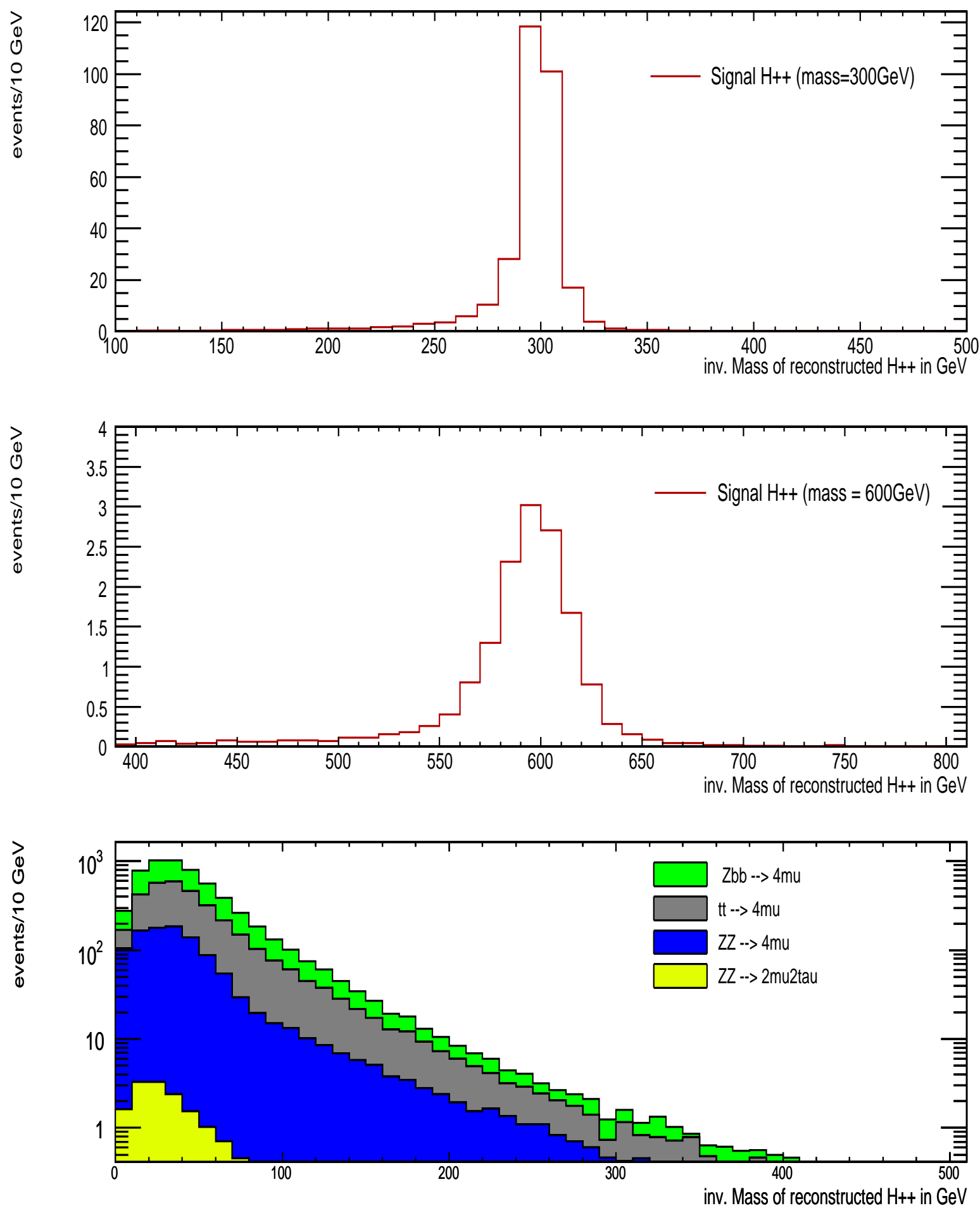

Figure 6: The invariant mass distribution of of the signal sample $m_{H^{ \pm \pm}}=300 \mathrm{GeV}$, of the signal sample $m_{H^{ \pm \pm}}=$ $600 \mathrm{GeV}$ and of the four backgrounds. 


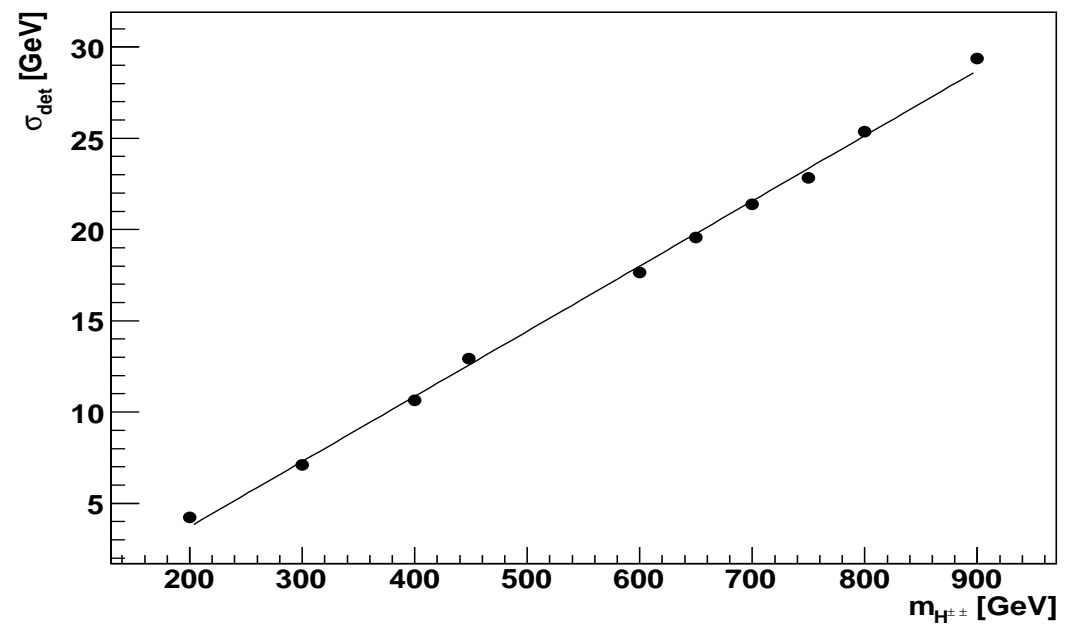

Figure 7: Mass resolution as a function of $m_{H^{ \pm \pm}}$.

\section{Offline Event Selection}

Very few cuts were used in this study in order to keep the signal efficiency as high as possible. Because of the same-charge muon final state, the reconstructed invariant mass of the signal can be clearly distinguished from the background, even before any offline selection. The offline event selection cuts is described in the next paragraphs.

\subsection{The Number of Reconstructed Muons}

The first step in the offline event selection is to require four muons with $p_{T}>8 \mathrm{GeV}$ and $|\eta|<2.1$. The requirements on $\eta$ and $p_{T}$ are direct consequences of the muon trigger requirements (Section 5). The efficiency of this selection is shown in Table 5 for the background data samples and for the $m_{H^{ \pm \pm}}=300 \mathrm{GeV}$. This cut reduces significantly the amount of background.

Table 5: Selection efficiency for four reconstructed muons with $p_{T}>8 \mathrm{GeV}$ in $|\eta|<2.1$ for a signal datasample $\left(m_{H^{ \pm \pm}}=300 \mathrm{GeV}\right)$ and the background datasamples; errors are statistical only

\begin{tabular}{|l|c|cccc|}
\hline & $\begin{array}{c}\text { Signal } \\
m_{H^{ \pm}}=300 \mathrm{GeV}\end{array}$ & $Z \bar{Z} \rightarrow 4 \mu$ & $Z \bar{Z} \rightarrow 2 \mu+2 \tau$ & $Z b b \rightarrow 4 \mu$ & $t \bar{t} \rightarrow 4 \mu$ \\
\hline $4 \mu$ reconstruction efficiency in $\%$ & $76 \pm 1$ & $26.5 \pm 0.2$ & $22.6 \pm 0.6$ & $12.9 \pm 0.1$ & $30.2 \pm 0.3$ \\
\hline
\end{tabular}

\subsection{Impact Point}

Events with muons from different primary collisions, i.e. two or three muons from one collision and one or two from another, may become a significant source of background because of the much higher rates compared to genuine $4 \mu$ processes. For each muon in an event the Impact Point (Section 6.1) is determined. The longitudinal distances, $\Delta z_{I P S}$, between the Impact Points of all muons are calculated for each event. The largest $\Delta z_{I P S}$ is required to be smaller than $0.05 \mathrm{~cm}$. Figure 8 shows the distribution of the largest distance $\Delta z_{I P S}$ between muons generated in the decay of the doubly charged Higgs bosons for the signal sample $m_{H^{ \pm \pm}}=300 \mathrm{GeV}$. This distance is much smaller than the distribution of the z-coordinate for the Impact Point of all muons in the same data plotted in figure 9. The gaussian width of the distribution of the z-coordinate is $5.3 \pm 0.1 \mathrm{~cm}$ and it corresponds to the longitudinal size of the luminous region of the LHC beam of about $5 \mathrm{~cm}$. Therefore, with a probability greater than $99 \%$, the distance between two different collision vertices is greater than $0.05 \mathrm{~cm}$. The signal efficiency of this cut is roughly $96 \%$ as can be seen in Table 6 . This cut also reduces the $Z \bar{b} b$ and the $t \bar{t}$ background by roughly a factor 2 , due the displaced Impact Points of the muons generated in $b$ decays. 

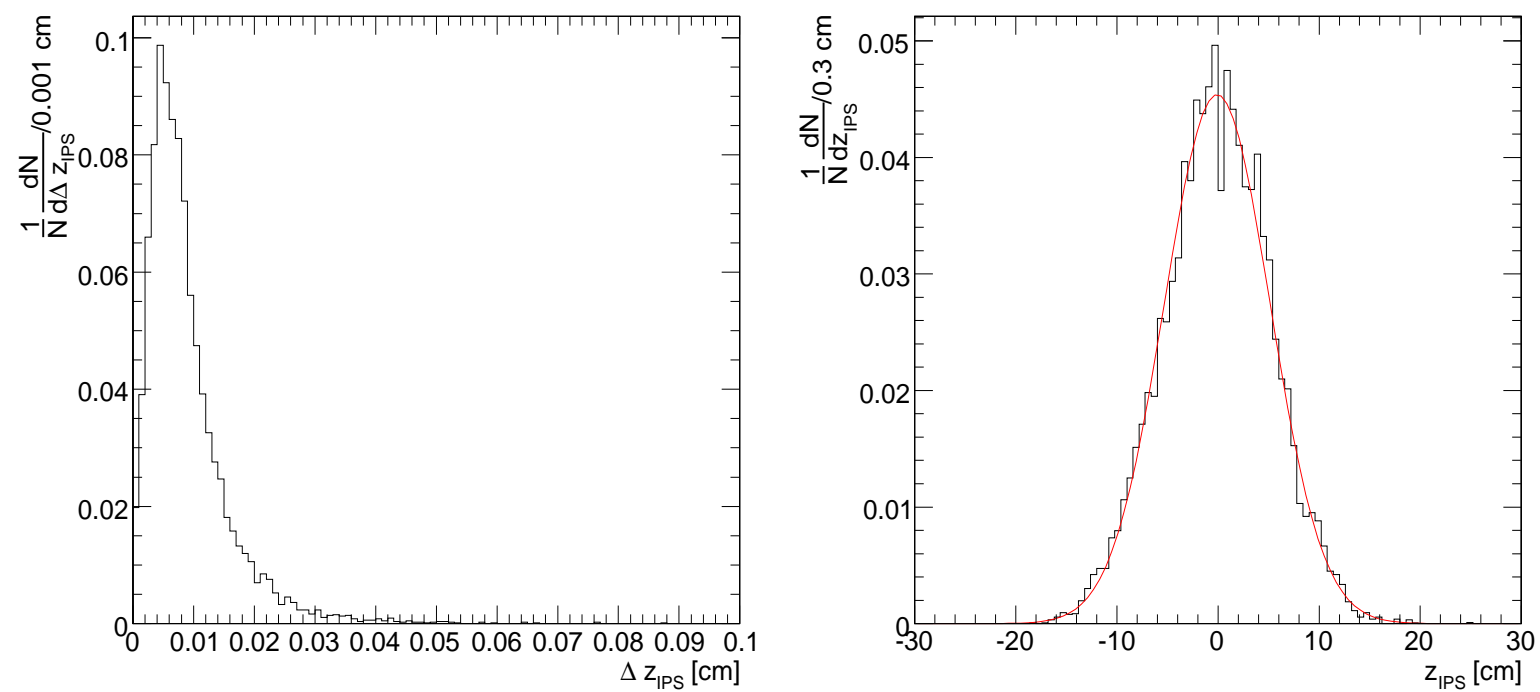

Figure 8: The biggest $\Delta z_{I P S}$ between muons gener-

Figure 9: $z_{I P S}$ of all muons generated for $L=$ ated in the decay of doubly charged Higgs bosons for $L=10 \mathrm{fb}^{-1}$ and $m_{H^{ \pm \pm}}=300 \mathrm{GeV}$

\section{Results}

Table 6 and Table 7 show the NLO production cross-section without any forced decay, the cross-section times branching ratio times pre-selection efficiency and the cross-section times branching ratio times efficiency after each stage of the online and offline event selection. Table 6 shows these values for each of the background samples. Table 7 show these values for signal samples with doubly charged Higgs masses of 300, 600 and $800 \mathrm{GeV}$.

Table 6: Production cross-section (NLO), cross-section times branching ratio times pre-selection efficiency and cross-section times branching ratio times efficiency after each stage of the online and offline event selection for background events. All values are expressed in fb. Errors are statistical only.

\begin{tabular}{|c|c|c|c|c|}
\hline & $\mathrm{t} \overline{\mathrm{t}}$ & $\mathrm{Zb} \overline{\mathrm{b}}$ & $\mathrm{ZZ} / \gamma^{\star} \rightarrow 4 \mu$ & $\mathrm{ZZ} / \gamma^{\star} \rightarrow 2 \mu+2 \tau$ \\
\hline Production cross-section (NLO) [fb] & $840 \cdot 10^{3}$ & $278 \cdot 10^{3}$ & 153 & 212 \\
Pre-Selection: $\sigma \cdot \mathrm{BR} \cdot \epsilon[\mathrm{fb}]$ & $232 \pm 1$ & $289.8 \pm 0.9$ & $87.4 \pm 0.3$ & $1.63 \pm 0.02$ \\
\hline Level-1 trigger [fb] & $232 \pm 1$ & $289 \pm 1$ & $87.3 \pm 0.3$ & $1.63 \pm 0.02$ \\
High Level Trigger [fb] & $149 \pm 1$ & $195 \pm 1$ & $69.7 \pm 0.3$ & $1.10 \pm 0.01$ \\
\hline $4 \mu$ reconstructed $\left(\mathrm{p}_{T}>8 \mathrm{GeV}|\eta|<2.1\right)[\mathrm{fb}]$ & $45.1 \pm 0.4$ & $25.1 \pm 0.3$ & $18.5 \pm 0.1$ & $0.25 \pm 0.01$ \\
Impact Point Cut [fb] & $22.8 \pm 0.3$ & $13.1 \pm 0.2$ & $16.9 \pm 0.1$ & $0.22 \pm 0.01$ \\
\hline
\end{tabular}

Figure 10 shows the invariant mass of the reconstructed doubly charged Higgs boson before and after the application of the online and offline selection, for signal events $m_{H^{ \pm \pm}}=300 \mathrm{GeV}$ and $m_{H^{ \pm \pm}}=600 \mathrm{GeV}$. The chosen bin size is $20 \mathrm{GeV}$, this bin size is comparable to the detector resolution of the doubly charged Higgs boson mass $\sigma_{\text {det }}$ for $m_{H^{ \pm \pm}}=600 \mathrm{GeV}$ (Section 6.2).

Even before the offline selection, the Higgs mass is clearly distinguishable from the background. Nevertheless loosening the offline requirements would not make any good sense. The signal efficiency is mostly affected by requiring the presence of four reconstructed muons. If three or two muons were required instead, the two or three muon backgrounds would reduce the signal significance. 
Table 7: Production cross-section (NLO), cross-section times branching ratio times pre-selection efficiency and cross-section times branching ratio times efficiency after each stage of the online and offline event selection, for $m_{H^{++}}=300,600,800 \mathrm{GeV}$ All values are expressed in fb. Errors are statistical only.

\begin{tabular}{|c|c|c|c|}
\hline & $m_{H^{ \pm \pm}}=300 \mathrm{GeV}$ & $m_{H^{ \pm \pm}}=600 \mathrm{GeV}$ & $m_{H^{ \pm \pm}}=800 \mathrm{GeV}$ \\
\hline Production cross-section (NLO) [fb] & 19.6 & 0.909 & 0.201 \\
Pre-Selection: $\sigma \cdot \mathrm{BR} \cdot \epsilon[\mathrm{fb}]$ & $17.4 \pm 0.3$ & $0.85 \pm 0.02$ & $0.190 \pm 0.004$ \\
\hline Level-1 trigger [fb] & $17.3 \pm 0.3$ & $0.85 \pm 0.02$ & $0.190 \pm 0.004$ \\
High Level Trigger [fb] & $17.1 \pm 0.3$ & $0.83 \pm 0.02$ & $0.188 \pm 0.004$ \\
\hline $4 \mu$ reconstructed $\left(\mathrm{p}_{T}>8 \mathrm{GeV}|\eta|<2.1\right)[\mathrm{fb}]$ & $13.0 \pm 0.2$ & $0.70 \pm 0.02$ & $0.158 \pm 0.003$ \\
Impact Point Cut [fb] & $12.5 \pm 0.2$ & $0.67 \pm 0.02$ & $0.153 \pm 0.003$ \\
\hline
\end{tabular}
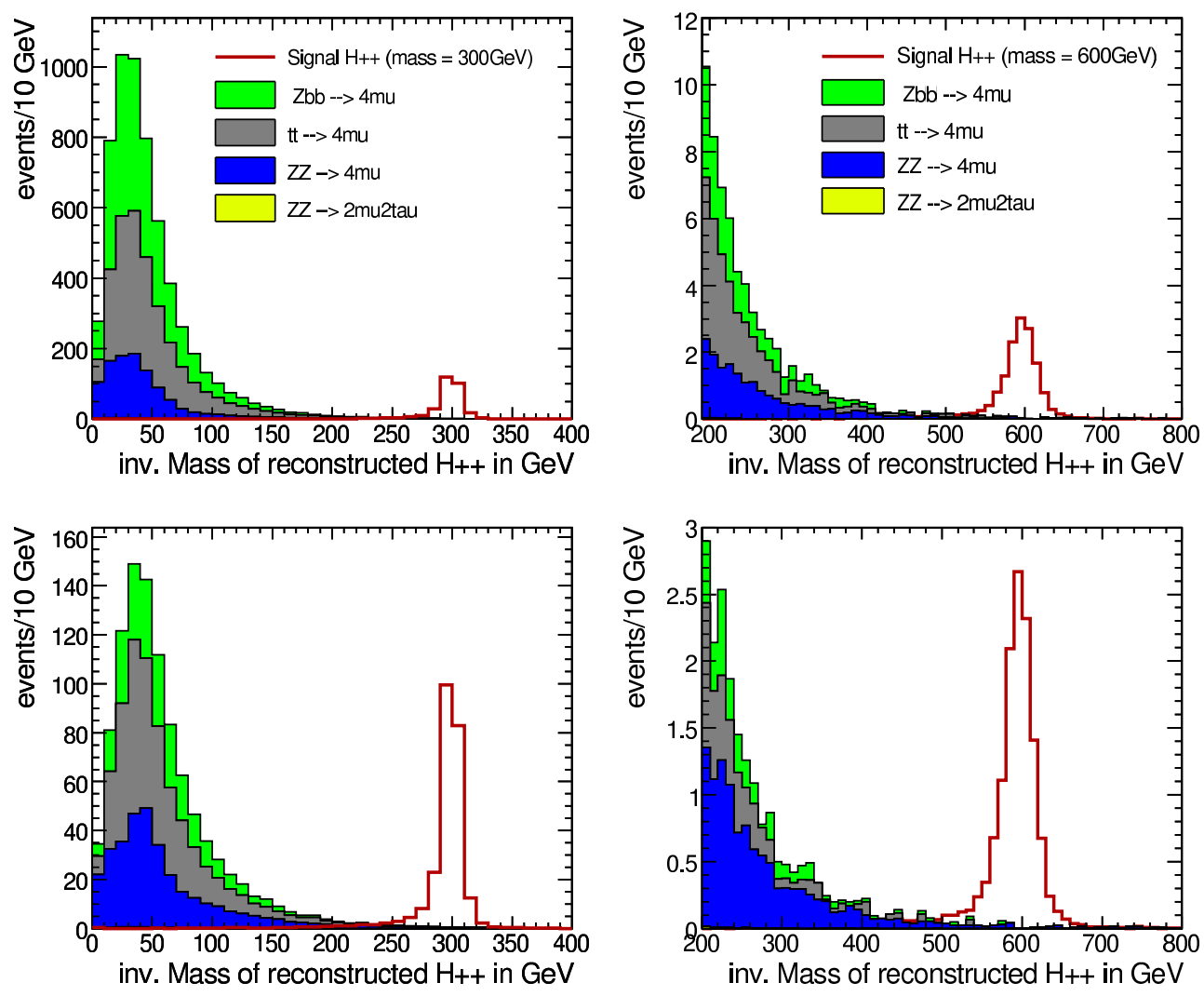

Figure 10: reconstructed invariant mass of $\mathrm{H}^{ \pm \pm}$candidates, (top) after online selection, (bottom) after offline selection

\section{Statistical Interpretation}

\subsection{The $\mathrm{CL}_{s}$ Method}

In order to understand up to which $H^{ \pm \pm}$masses a signal for new physics can be detected or excluded, the invariant mass spectrum is subject to statistical analysis. The hypothesis test is performed using the $\mathrm{CL}_{s}$-method [22, 21, 20], which takes into account the form of the signal peak without requiring any cut on the invariant mass. It interprets 
each of the $\mathrm{N}$ bins of the invariant mass spectrum in Figure 10 as a result of $\mathrm{N}$ independent Poisson counting experiments with the probability distribution

$$
P(\mu ; n)=\frac{\mu^{n}}{n !} \cdot e^{-\mu}
$$

$P(\mu ; n)$ reflects the probability to obtain the result $n$ in a counting experiment if $\mu$ is expected.

These two probabilities, $\mathrm{P}(\mu=s+b ; n)$ and $\mathrm{P}(\mu=b ; n)$, can be used to construct a statistical estimator for the significance of a signal. The discrimination variable used in the $C L_{s}$ method can be calculated for each bin as:

$$
Q_{i}\left(m_{H^{ \pm \pm}}\right)=\frac{P\left(\mu=s_{i}\left(m_{H^{ \pm \pm}}\right)+b_{i} ; n_{i}\right)}{P\left(\mu=b_{i} ; n_{i}\right)},
$$

where $s_{i}()$ is the number of signal events in bin $i$, which is a function of the doubly charged Higgs boson mass $m_{H^{ \pm \pm}}$, and $b_{i}$ is the number of background events in bin $i$. Both numbers are normalized to an integrated luminosity of $10 \mathrm{fb}^{-1}$. The combination of all $\mathrm{N}$ "per bin counting experiments" for a chosen doubly charged Higgs boson mass $m_{H^{ \pm \pm}}$leads to:

$$
-2 \ln Q\left(m_{H^{ \pm \pm}}\right)=-2 \sum_{i=1}^{N}\left[n_{i} \ln \left(1+\frac{s_{i}}{b_{i}}\right)-s_{i}\right]=2 s_{t o t}-2 \sum_{i=1}^{N} n_{i} \ln \left(1+\frac{s_{i}}{b_{i}}\right)
$$

where $n_{i}$ is once the number of background events in bin $\mathrm{i}$ and once the number of signal plus background events. Figure 11 shows the distribution of -2lnQ for various doubly charged Higgs boson masses for the background only hypothesis and the signal plus background hypothesis. Where $n_{i}$ is once the number of background events in bin $\mathrm{i}$ and once the number of signal plus background events.

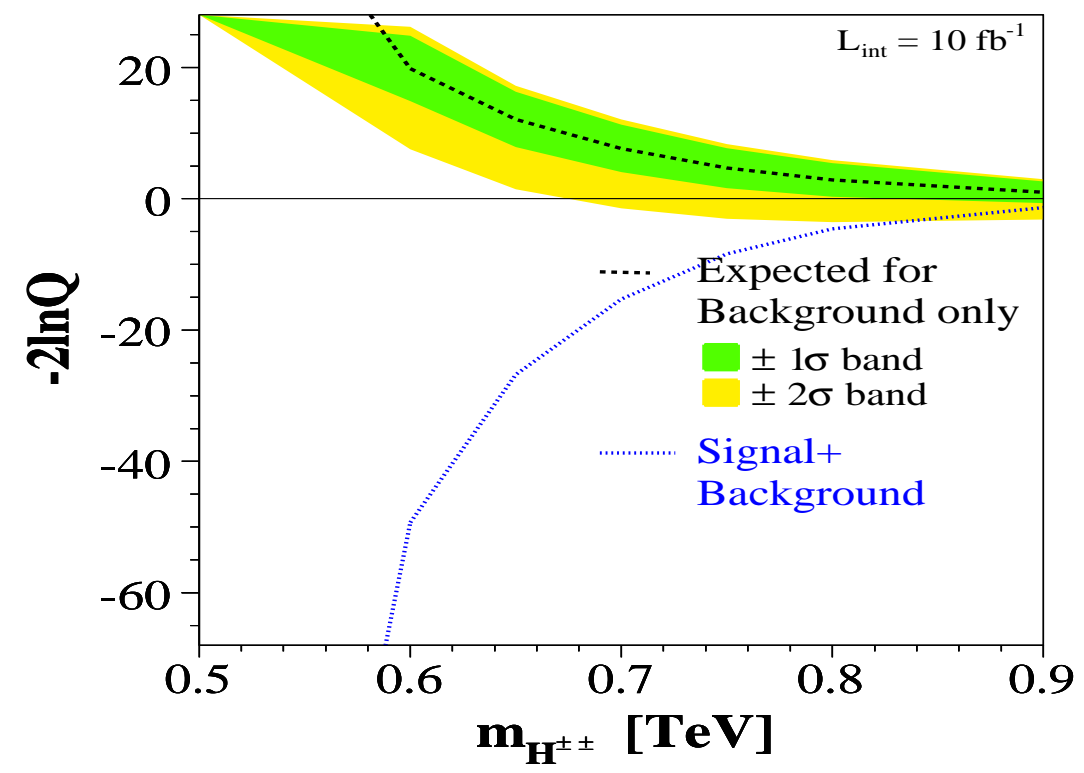

Figure 11: -2lnQ as a function of the doubly charged Higgs boson mass for the background only hypothesis and for the signal plus background hypothesis

The determination of the signal significance is performed by generating numerous "pseudo-experiments": for each bin in the invariant mass spectrum. Poisson distributed random numbers are generated once using the mean value $\mu=s_{i}+b_{i}$ and once using $\mu=b_{i}$ to generate different $n_{i}$ (Equation 7).

The Equation 7 is evaluated for each "experiment" obtaining two almost Gaussian shaped distributions for each doubly charged Higgs boson mass centered at the $-2 \ln Q$ of the signal plus background expectation and the background only expectation, respectively (Figure 12).

For the determination of the different confidence levels (CL) for each hypothesis, the distributions of the achieved pseudo-statistic are integral-normalised and defined as $\mathcal{P}_{\mathrm{s}+\mathrm{b}}(x)$ and $\mathcal{P}_{\mathrm{b}}(x)$ using $x:=-2 \ln Q$, respectively. To 

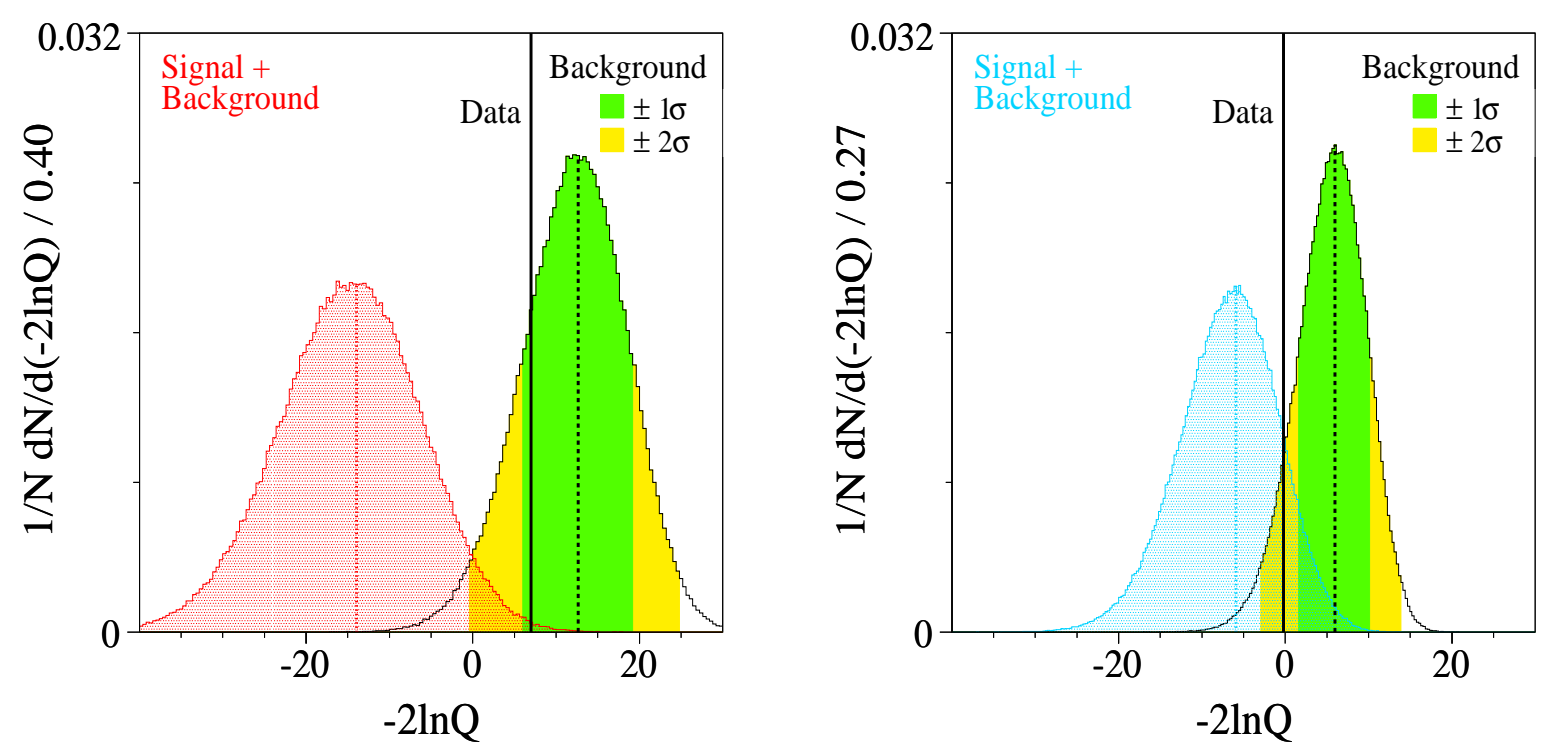

Figure 12: Typical distribution of the variable $-2 \ln Q$ for different hypothetical signals. For the Gaussian-like distributions the corresponding $\sigma$-bands are shown: 68\% (95\%) of the pseudo-experiments are within one (two) standard deviations around the mean. The $-2 \ln Q$ value for a hypothetical measurment "Data" is included in these plots.

decide, if the hypothesis of having measured background only is true, the integral

$$
C L_{b}=\int_{X_{0}}^{\infty} \mathcal{P}_{b}(x) d x
$$

is investigated, where $X_{0}$ is the value of $-2 \ln Q$ from an experiment (real data) or the mean value of the signal plus background $-2 \ln Q$ distribution (simulated data). A small value of $1-C L_{b}$ implies a small probablity for the experimental data to be in agreement with the background only expectation.

In the same way one can test the significance of an excess of signal above the background, using

$$
C L_{s+b}=\int_{X_{0}}^{\infty} \mathcal{P}_{s+b}(x) d x
$$

Assuming a Gaussian distribution for both $\mathcal{P}_{\mathrm{s}+\mathrm{b}}(x)$ and $\mathcal{P}_{\mathrm{b}}(x)$ a possible signal can be distinguished from the background if the signal plus background expectation exceeds the background only expectation by more than $5 \sigma$.

$$
1-C L_{b} \leq 2.85 \cdot 10^{-7}
$$

If no signal will be discovered an exclusion limit can be calculated by convention with $95 \%$ confidence. Thus, the probability for excluding the presence of signal is less than $5 \%$, which can be mathematically described by

$$
C L_{s}:=\frac{C L_{s+b}}{C L_{b}}<0.05 .
$$

The statistical error on the confidence levels can be extracted from $P_{s+b}$ and $P_{b}$. The width $\sigma$ of the distributions is determined. The integration border $X_{0}$ is varied by $\pm 1 \sigma$ and $\pm 2 \sigma$. The resulting variations in the confidence levels are taken as statisticals errors.

\subsection{Background Event Statistic}

For invariant masses greater than $\sim 500 \mathrm{GeV}$ nearly no background has been simulated, as shown in Fig.13 and Fig.14. The $C L_{s}$ significance estimator cannot take into account bins without background. To calculate the signal significance for doubly charged Higgs boson masses greater than $500 \mathrm{GeV}$, the empty bins had to be filled by an estimated background distribution. Two possible solutions are: 
1. The empty bins are filled, using Poisson statistics:

The $\mathrm{N}$ bins in the invariant mass spectrum are assumed to be the result of $\mathrm{N}$ independent Poisson counting experiments. If a backgrounds has no entries in a certain bin, the mean of the corresponding Poisson distribution has, at $95 \%$ confidence level, to be $\leq 3$ events. To be conservative the bin is filled with 3 background events which are multiplied by the corresponding scale factor. The scale factor increases the total number of generated events to the number of events expected for the studied integrated luminosity. This procedure is repeated for each background. Figure 13 shows the invariant mass spectrum before and after the bins have been filled.

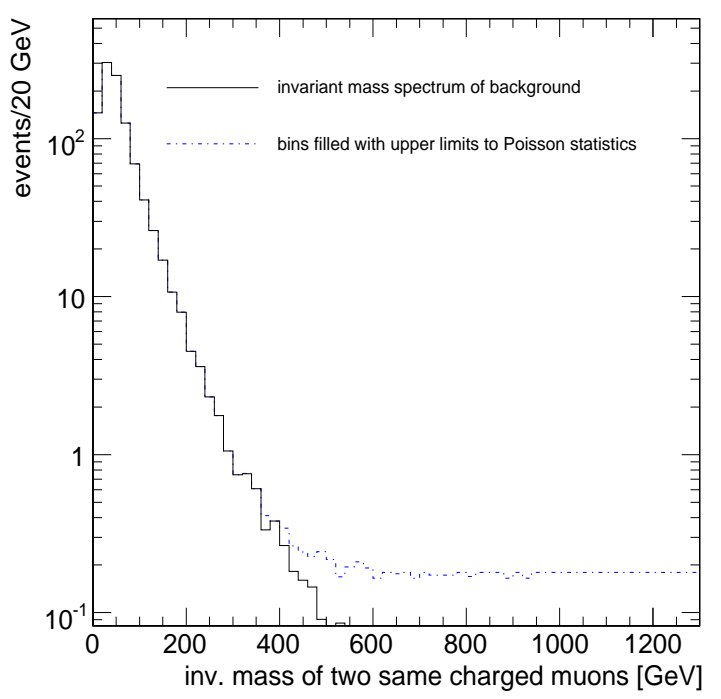

Figure 13: Distribution of the invariant reconstructed $H^{ \pm \pm}$mass for all background events, before and after the empty bins have been filled with upper limits to the Poisson statistics

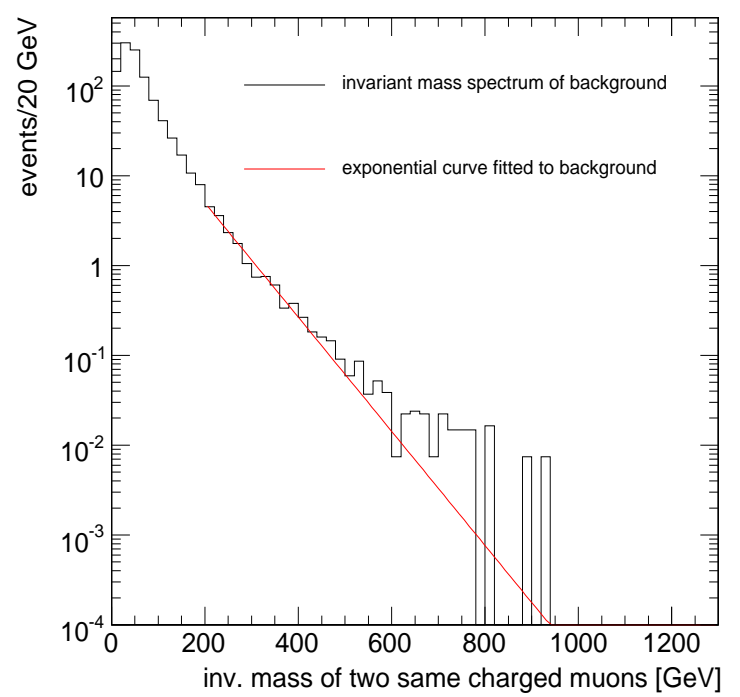

Figure 14: Distribution of the invariant reconstructed $H^{ \pm \pm}$mass for all background events and the estimated distribution of the invariant mass following an exponential decreasing curve

2. An exponential decreasing curve is fitted to the reconstructed invariant mass spectrum of the background:

The mass distribution reconstructed from the decay products of a non-resonant state typically follows (at least locally) a decaying exponential or, more precisely the tail of a Landau distribution. By fitting an exponential curve to the invariant mass spectrum the number of expected events in the different bins can be estimated. The curve is fitted to the invariant mass spectrum for masses larger than $200 \mathrm{GeV}$, because below this limit enough background events have been generated and above this limit the invariant mass distribution follows an exponential decreasing function. The fitted curve and the errors on the fit are:

$$
\exp \left[4.5( \pm 1)-1.5 \cdot 10^{-2}\left( \pm 4 \cdot 10^{-3}\right) \cdot m_{i n v}\right],
$$

where $m_{i n v}$ is the reconstructed invariant mass in GeV. Figure 14 shows the generated and reconstructed distribution of the invariant mass and the exponential curve fitted to this distribution. This method depends strongly on the mass region chosen for fitting the curve.

Both methods have their advantages and disadvantages. The more conservative method 1 is chosen and applied in this work. The exclusion and discovery limits obtained with method 2, are taken as an estimate of the systematical error on the number of background events.

\subsection{Discovery Limit}

Figure 15 shows the value of $1-C L_{b}$ for the different doubly charged Higgs boson masses. For a doubly charged Higgs boson mass smaller than $650 \mathrm{GeV}$ the signal plus background expectation will exceed the background only expectation by more than $5 \sigma$. Up to this mass a possible existing signal in the four muon decay channel could be 
If the less conservative method 2 described in Section 9.2 is used, the exclusion and discovery limit improve respectively to $800 \mathrm{GeV}$ and $700 \mathrm{GeV}$. This variation in the exclusion and discovery mass limit, for the different methods, can be regarded as systematic uncertainty, due to the lack of simulated background events for higher doubly charged Higgs boson masses.

\section{Conclusions}

A feasibility study for discovery of pair-produced doubly charged Higgs bosons with the full detector simulation has been presented. A branching ratio of $100 \%$ into muons has been assumed. The doubly charged Higgs boson mass, which is the only free parameter for pair-produced doubly charged Higgs bosons, has been investigated in the range 100-800 GeV. All Standard Model background processes have been taken into account. According to the low luminosity phase of the LHC $\left(\mathcal{L}=2 \cdot 10^{33} \mathrm{~cm}^{-2} \mathrm{~s}^{-1}\right)$, on average 3.5 pileup events have been mixed with the signal and background samples. For both signal and background NLO cross sections have been used.

It has been shown, that pair-produced doubly charged Higgs bosons with a branching of $100 \%$ into muons, can be discovered $(5 \sigma)$ with an integrated luminosity of $10 \mathrm{fb}^{-1}$ in the mass range of $100-650 \mathrm{GeV}$. If no signal is visible at CMS, an exclusion limit at $95 \% \mathrm{CL}$ of $\mathrm{m}\left(\mathrm{H}^{ \pm \pm}\right)=760 \mathrm{GeV}$ can be set. This exceeds the current exclusion limit for this channel $\mathrm{m}\left(\mathrm{H}^{ \pm \pm}\right) \leq 136 \mathrm{GeV}$, set by $\mathrm{CDF}$ at Tevatron Run II, by $624 \mathrm{GeV}$.

Systematic uncertainties resulting from higher order cross sections, parton density functions and scale dependencies have been calculated for the signal by Spira and Muehlleitner [6]. The systematic uncertainties of the backgrounds have been taken from studies of the Standard model process $H \rightarrow Z Z \rightarrow 4 \mu$, which has the same backgrounds and is studied in detail as a benchmark process for the CMS detector.

\section{Acknowledgments}

We would like to thank all the members of the CMS collaboration who contributed to the software used within this analysis and supported this study. We would like to thank our referees Pablo Garcia Abia, Guenakh Mitselmakher, Nancy Marinelli and Roberto Tenchini. We are especially grateful to Alexandre Nikitenko, Alexey Drozdetskiy, Andreas Nowack, Marc Zoeller, Mario Kadastik, Martti Raidal, Michael Spira, Norbert Neumeister, Sami Lehti and Torbjorn Sjostrand for their discussions and assistance. 


\section{References}

[1] Phys. Rev.Lett. 81 (1998) 1562[arXiv:hep-ex/987003], Super Kamiokande Collaboration, Y.Fukuda et al., Evidence for oscillation of solar neutrinos,

[2] The CMS Collaboration, ”CMS Technical Proposal”, CERN/LHCC 94-38, LHCC/P1, 1994

[3] Phys. Letter. B539,179(2002)[arXiv:hep-ex/0205075], Super Kamiokande Collaboration, S.Fukuda et al., Determination of solar neutrino oscillation parameters using 1496 days of Super-Kamiokande-I data

[4] Phys. Rev. Letter.93,221802(2003), CDF Collaboration, D.Acosta et al., Search for Doubly-Charged Higgs Bosons Decaying to Dileptons in pp Collisions at $\sqrt{s}=1.96 \mathrm{TeV}$.

[5] hep-ph/9606311, K.Huitu et al., Doubly charged Higgs at the LHC.

[6] hep-ph/0305288, M.Muehlleitner and M.Spira, A Note on Doubly-Charged Higgs Pair Production at Hadron Colliders.

[7] http://hcp-2005.web.cern.ch/HCP-2005 F.Maltoni Theoretical Issues and Aims at the Tevatron and LHC, talk on HCP2O05

[8] http://indico.cern.ch/conferenceDisplay.py?confId=a061694 P.Bartalini, H. Stenzel and A. Sherstnev, presented by A. Korytov at the $H \rightarrow Z Z^{(*)} \rightarrow 4 l$ meeting CMS Note in preparation

[9] hep-ph/0506176, A.G. Akeroyd and M. Aoki, Single and Pair Production of Doubly Charged Higgs Bosons at Hadron Colliders.

[10] Phys. Rev.D34(1986)909, R.Mohapatra, Limits on the mass of the right-handed Majorana neutrino.

[11] report LU-TP-01-21, Aug 2001[arXiv:hep-ph/9198264], T.Sjostrand, L.Lonnblad and S.Mrenna, PYTHIA 6.2 Physics and Manual.

[12] arXiv:hep-ph/9908288, Aug 1999,, A.Pukhhov, E.Boos, M.Dubinin et al

[13] http://cmsdoc.cern.ch/cmsoo/projects/CMKIN CMS Interface for Event Generators

[14] http://cmsdoc.cern.ch/orca/, CMS OO Reconstruction-Users's Guide and Reference Manual.

[15] http://cmsdoc.cern.ch/cms/aprom/www/top/aprom.html, CMS OO Reconstruction-Users's Guide and Reference Manual.

[16] arXiv:hep-ph/0105226 J.M.Campbell W/Z + B anti-B/jets at NLO using the Monte Carlo MCFM

[17] arXiv:hep-ph/0208156 F.Maltoni and T.Steltzer MadEvent:Automatic Event Generation with MadGraph, JHEP 0302(2003)027

[18] CERN/LHCC 2002-26(2002), CMS TDR 6.2; CMS Collaboration The TriDAS Project Technical Design Report, Volume2: Data Acquisition and High Level Trigger.

[19] CERN/LHCC 97-32, CMS TDR 3; CMS Collaboration CMS MUON Technical Design Report, chapter 2.3.

[20] Prepared for Workshop on Confidence Limits, Geneva, Switzerland (2000) A. L. Read, "Modified Frequentist Analysis of Search Results (The CLs Method)",

[21] Proceedings of 14th Rencontres de Physique de la Valle d'Aoste: Results and Perspectives in Particle Physics, La Thuile, Valle d'Aoste, Italy (2000) E. Gross and A. L. Read, "Prospects for Standard Model Higgs Search in the LEP 2000 Run",

[22] J. Phys. G28 (2002) 2693-2704 A. L. Read, "Presentation of Search Results: The $C L_{s}$ Technique”,

[23] Oxford University Press, New York, 1998 G. Cowan, "Statistical Data Analysis",

[24] Springer, New York, 1997 S. Brandt, "Statistical and Computational Methods in Data Analysis",

[25] L3 Internal Note 2066, 1997 A. Favara and M. Pieri, "Optimal Statistical Analysis of Search Results",

[26] CMS NOTE in preparation M.Aldaya, P.Arce, J.Caballero, B. de la Cruz, P. Garcia-Abia, J.M. Hernandez, M.I.Josa, E.Ruiz, Search for the Standard Model Higgs boson in the $H \rightarrow Z Z \rightarrow 4 \mu$ decay channel using a massindependent analysis 